\title{
Electrode Passivation in the Electrocoagulation Process
}

\author{
Gilberto B. Rios,* Facundo Almeraya, Ma. Teresa A. Herrera \\ Centro de Investigación en Materiales Avanzados Miguel de Cervantes 120. Complejo \\ Ind. Chihuahua. CP 31109, Chihuahua, Chihuahua. México.
}

Received 15 February 2004; accepted in revised form 12 December 2004

\begin{abstract}
One of the main limiting factors of the electrocoagulation, as a process of treatment for remotion of contaminants from residual waters, is not knowing the degree of interaction of some variables which could significantly affect the system. The electrode passivation is one of them once its presence limits the emission of metallic ions and consequently the treatment time is increased as well as cost, and remotion efficiency is decreased. In the present work potentiodynamic and potentiostatic curves were used to observe the behaviour of current density vs. $\mathrm{pH}$, electrical conductivity, distance between electrodes, auxiliary electrode area and applied potential difference. We found out that all the above variables influence the current density, that passivation is at $50 \mathrm{mV}$, independently of all the other variables, and that at $2000 \mathrm{mV}$, only is observed when $\mathrm{pH}$ $=2$ and high electrical conductivities.
\end{abstract}

Keywords: potentiostatic and potentiodynamic curves, current density, residual water.

\section{Introducción}

La electrocoagulación, es una tecnología que en la última década ha aumentado su uso en América del Sur y Europa con resultados muy prometedores en el tratamiento de agua residual y potable [1,2]. Algunas de sus ventajas son: facilidad de operación, fabricación nacional, no requiere la aplicación de químicos, produce hasta un 50\% menos lodos que la coagulación convencional y posee una elevada eficiencia en la remoción de contaminantes [3, 4]. Dentro de sus principales desventajas se encuentra que los mecanismos de la electrocoagulación no han sido aún claramente entendidos y con frecuencia se recurre a pruebas de ensayo - error para adaptar el sistema a una determinada

\footnotetext{
${ }^{*}$ Corresponding author. E-mail address: gilberto.badillo@cimav.edu.mx
} 
calidad de influente y/o efluente [5]; una de estas variables que afecta significativamente tanto en las eficiencias de remoción como en los costos de operación es la pasivación [2, 3].

Pasivación es la propiedad que presentan determinados metales y aleaciones de permanecer prácticamente inertes en determinados medios, en los que, de acuerdo con la termodinámica, deberían comportarse como activos y por lo tanto disolverse con velocidades altas a través de un mecanismo de corrosión electroquímico. La pasivación puede ser consecuencia de la formación de una capa de pequeño espesor de productos oxidados sobre la superficie del electrodo, o bien, por la formación de capas monoatómicas de $\mathrm{O}_{2}$ sobre la superficie del cátodo [6]. Cada metal presenta un determinado umbral de potencial y $\mathrm{pH}$ en donde los iones que entran a la solución pueden ser solubles y en tal caso no se presenta la pasivación; sin embargo, cuando los iones liberados no son solubles, la capa de hidróxidos puede presentarse con facilidad [7].

La pasivación de un electrodo puede demostrarse con una curva de corriente de potencial anódico. Cuando se imponen potenciales mayores al potencial de equilibrio metal - medio, se produce la oxidación, obteniéndose registros de intensidad de corriente que crecen exponencialmente al aumentar el potencial; si el electrodo es pasible para un determinado valor de potencial, se produce una caída significativa en la intensidad de corriente. El factor tiempo de operación participa activamente en la formación de la capa pasiva, afectando los resultados obtenidos en las curvas potenciodinámicas y por lo tanto, es necesario complementar el estudio con curvas potenciostáticas para determinar a que potenciales y en que tiempo de tratamiento se presentan o no los efectos de la pasivación.

Además del potencial, diferentes estudios marcan el efecto significativo de algunas otras variables sobre la eficiencia de remoción de contaminantes, como son $\mathrm{pH}[8,9]$ (que influye tanto en el proceso electrolítico como en la coagulación - floculación), conductividad eléctrica (que influye en la resistencia al paso de la corriente), distancia entre electrodos (que influye también en la resistencia al paso de la corriente), el área del electrodo de trabajo con respecto 
al electrodo auxiliar (que afecta a la mayor o menor disponibilidad de iones $\mathrm{H}^{+}$

u $\left.\mathrm{OH}^{-}[7]\right)$, y que a su vez, pudieran influir en el tiempo que tarda en formarse la capa pasiva.

Las variables antes mencionadas han sido estudiadas en forma independiente observando su efecto en la remoción de contaminantes, cuya eficiencia está directamente relacionada con la cantidad de iones metálicos producios electroquímicamente; este estudio muestra el efecto, independiente y en interacción, de estas variables en la densidad de corriente, ya que ésta última es quien determina la cantidad de iones metálicos que se añaden al electrolito, y el efecto de la pasivación de los electrodos en la interpretación de resultados.

\section{Desarrollo experimental}

Las curvas potenciodinámicas se desarrollaron empleando equipo Autotafel ACM Instruments y para las curvas potenciostáticas un Potenciostato/Galvanostato/ZRA Gill 8 de ACM Instruments. Para ambos estudios se emplearon, además, un conductividimetro Cornning 441, pHmetro Denver Instruments 250; electrodo de referencia calomelano (ECS), puente salino, electrodo de trabajo de hierro de bajo carbono embebido en resina y pulido, con área expuesta de $1 \mathrm{~cm}^{2}$; electrodo auxiliar de grafito con área expuesta de $2 \mathrm{~cm}^{2}, 0.5 \mathrm{~cm}^{2}, 1.125 \mathrm{~cm}^{2}$ que corresponden a los niveles máximo, mínimo y central, respectivamente, de un diseño de experimentos $2^{\mathrm{k}}$ con puntos centrales. Los electrodos y sensores se colocaron en el interior de la celda tal y como se muestra en la Fig. 1.

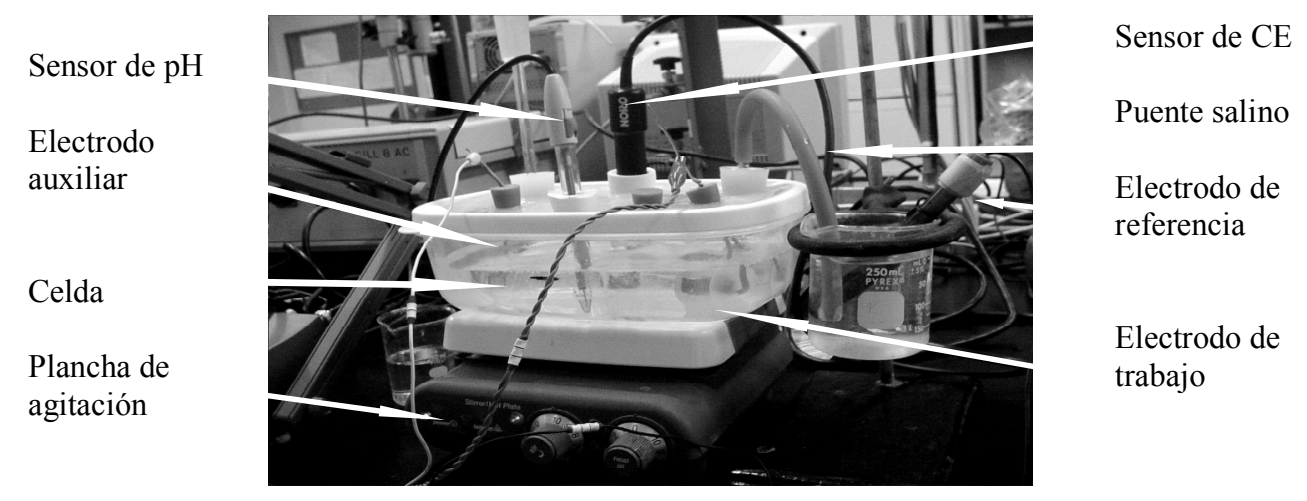

Figura 1. Vista lateral de la celda electroquímica con distribución de electrodos y sensores empleados. 
Las curvas potenciodinámicas y potenciostáticas se realizaron con los siguientes parámetros de prueba:

\section{Curvas potenciodinámicas}

- Diseño de experimentos $2^{4}$.

- Rango de barrido: -300 a $2500 \mathrm{mV}$ respecto al electrodo calomelano saturado (ECS) a partir del potencial de equilibrio.

- Velocidad de barrido: $60 \mathrm{mV} / \mathrm{min}$.

- Agitación turbulenta.

\section{Curvas potenciostáticas}

- Diseño de experimentos $2^{5-3}$ con puntos centrales y en tres bloques.

- Tiempo de 30 minutos.

- Agitación turbulenta.

El electrolito para ambos estudios es a base de agua destilada cuya conductividad eléctrica se ajustó con sulfato de sodio $\left(\mathrm{Na}_{2} \mathrm{SO}_{4}\right)$ y el pH se ajustó empleando ácido sulfúrico $\left(\mathrm{H}_{2} \mathrm{SO}_{4}\right)$ e hidróxido de sodio $(\mathrm{NaOH})$. Se emplearon los programas ORIGIN 5.0 y MINITAB 13.1 para el procesado de las gráficas y el análisis de resultados, respectivamente.

Las variables de estudio y sus niveles se describen en el cuadro 1 para curvas potenciodinámicas y en el cuadro 2 para curvas potenciostáticas. En ambos casos la variable de respuesta es la densidad de corriente.

\section{Resultados y discusión}

Dado que los resultados de un análisis estadístico de las curvas potenciodinámicas no son válidos para cada uno de los puntos de las curvas, sobre todo si estas se entrecruzan, el propósito de este trabajo al realizar curvas potenciodinámicas bajo un diseño de experimentos $2^{4}$, es el de analizar cualitativamente el comportamiento de estas curvas y tratar de identificar bajo que condiciones se 
puede presentar la pasivación, para posteriormente evitarlas en los estudios potenciostáticos. El análisis cualitativo se basó tanto en el comportamiento de las curvas como en las condiciones en que se realizaron cada una de ellas.

Cuadro 1. Variables de estudio y la respuesta obtenida en la densidad de corriente al final de cada curva potenciodinámica bajo un diseño de experimentos $2^{4}$.

\begin{tabular}{cccccc}
\hline Curva & $\mathrm{pH}$ & $\begin{array}{c}\mathrm{DE} \\
(\mathrm{cm})\end{array}$ & $\mathrm{Ac}$ & $\begin{array}{c}\mathrm{CE} \\
(\mathrm{mS} / \mathrm{cm})\end{array}$ & $\begin{array}{c}\mathrm{i} \\
\left(\mathrm{mA} / \mathrm{cm}^{2}\right)\end{array}$ \\
\hline 1 & 2.0 & 0.5 & 2.0 & 4.0 & 18.90 \\
2 & 12.0 & 0.5 & 2.0 & 4.0 & 12.90 \\
3 & 2.0 & 14.0 & 2.0 & 4.0 & 23.21 \\
4 & 12.0 & 14.0 & 2.0 & 4.0 & 11.48 \\
5 & 2.0 & 0.5 & 0.5 & 4.0 & 27.61 \\
6 & 12.0 & 0.5 & 0.5 & 4.0 & 54.48 \\
7 & 2.0 & 14.0 & 0.5 & 4.0 & 26.37 \\
8 & 12.0 & 14.0 & 0.5 & 4.0 & 11.05 \\
9 & 2.0 & 0.5 & 2.0 & 45.0 & 27.65 \\
10 & 12.0 & 0.5 & 2.0 & 45.0 & 54.89 \\
11 & 2.0 & 14.0 & 2.0 & 45.0 & 34.11 \\
12 & 12.0 & 14.0 & 2.0 & 45.0 & 70.68 \\
13 & 2.0 & 0.5 & 0.5 & 45.0 & 36.45 \\
14 & 12.0 & 0.5 & 0.5 & 45.0 & 68.09 \\
15 & 2.0 & 14.0 & 0.5 & 45.0 & 38.92 \\
16 & 12.0 & 14.0 & 0.5 & 45.0 & 53.50 \\
\hline
\end{tabular}

Al analizar la densidad de corriente final en cada curva se observa en el cuadro 1 que las curvas $6,10,12,14$, y 16 presentaron las más altas densidades de corriente coincidiendo todas ellas en un $\mathrm{pH} 12$; sin embargo, las curvas 2, 4 y 8 presentan las mismas condiciones de $\mathrm{pH}$ y la densidad de corriente es hasta un $84 \%$ menor, indicándonos la interacción de las variables de estudio ya que estas últimas curvas son quienes presentan las densidades de corriente más bajas. En general se observan mejores resultados a $\mathrm{CE}$ de $45 \mathrm{mS} / \mathrm{cm}$ y $\mathrm{pH} 12$ seguidos de curvas bajo la misma $\mathrm{CE}$ y pH 2.0 indicándonos una posible interacción de estas dos variables sobre la densidad de corriente. 
Cuadro 2. Densidades de corriente obtenidas en curvas potenciostáticas bajo un diseño de experimentos $2^{5-2}$ en 2 bloques y con puntos centrales.

\begin{tabular}{|r|r|r|r|r|r|r|r|r|}
\hline Curva & Blocks & $\mathbf{p H}$ & $\begin{array}{c}\text { DE } \\
\mathbf{c m}\end{array}$ & $\begin{array}{c}\text { Ac } \\
\mathbf{c m}^{\mathbf{2}}\end{array}$ & $\begin{array}{c}\mathbf{C E} \\
\mathbf{m S} / \mathbf{c m}\end{array}$ & $\begin{array}{c}\mathbf{E} \\
\mathbf{m V}\end{array}$ & $\begin{array}{c}\mathbf{5} \\
\text { minutos }\end{array}$ & $\begin{array}{c}\text { 30 } \\
\text { minutos }\end{array}$ \\
\hline 1 & 1 & 2 & 14.00 & 4.00 & 4 & 2000 & 24.668 & 24.302 \\
\hline 2 & 1 & 12 & 14.00 & 4.00 & 40 & 50 & 0.001 & 0.001 \\
\hline 3 & 1 & 2 & 0.50 & 0.50 & 40 & 50 & 1.050 & 0.462 \\
\hline 4 & 1 & 12 & 0.50 & 0.50 & 4 & 2000 & 10.506 & 10.512 \\
\hline 5 & 1 & 7 & 7.25 & 2.25 & 22 & 1025 & 49.178 & 50.019 \\
\hline 6 & 1 & 7 & 7.25 & 2.25 & 22 & 1025 & 48.744 & 49.001 \\
\hline 7 & 1 & 7 & 7.25 & 2.25 & 22 & 1025 & 49.002 & 51.160 \\
\hline 8 & 2 & 2 & 0.50 & 4.00 & 40 & 2000 & 194.186 & 0.228 \\
\hline 9 & 2 & 12 & 0.50 & 4.00 & 4 & 50 & 0.001 & 0.001 \\
\hline 10 & 2 & 2 & 14.00 & 0.50 & 4 & 50 & 0.213 & 0.050 \\
\hline 11 & 2 & 12 & 14.00 & 0.50 & 40 & 2000 & 24.457 & 25.077 \\
\hline 12 & 2 & 7 & 7.25 & 2.25 & 22 & 1025 & 46.796 & 46.796 \\
\hline 13 & 2 & 7 & 7.25 & 2.25 & 22 & 1025 & 48.123 & 48.975 \\
\hline 14 & 2 & 7 & 7.25 & 2.25 & 22 & 1025 & 50.021 & 51.142 \\
\hline
\end{tabular}

En la gráfica 1 se observa que todas las curvas presentaron el mismo comportamiento y no se observa pasivación en ninguna de ellas. Al analizar la curva 1 vs. la curva 5, se observa que se presentan mayores densidades de corriente en la curva 5 y la diferencia de las condiciones entre ellas es que en ésta última curva se tiene un área de electrodo auxiliar 2 veces menor que la empleada en la curva 1, indicando que en los estudios potenciostáticos posiblemente no se tendrá pasivación bajo estas condiciones de estudio y que se tendrán mayores densidades de corriente con el área del electrodo auxiliar 50\% menor que la del electrodo de trabajo. 


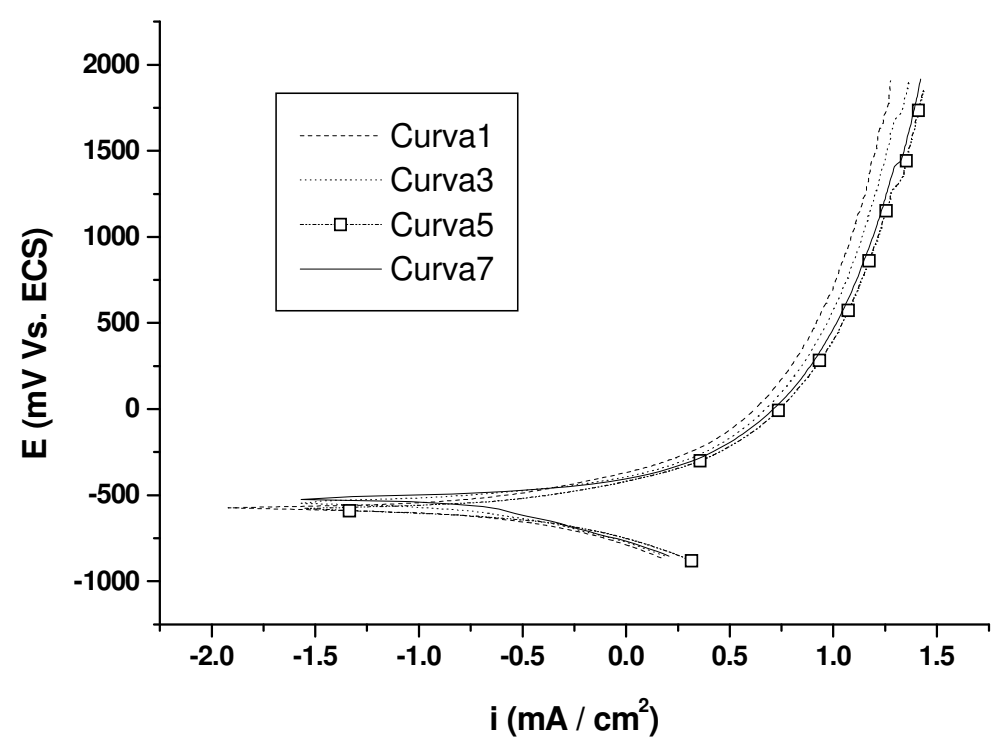

Gráfica 1. Curvas potenciodinámicas del diseño de experimentos $2^{4}$, bajo condiciones de $\mathrm{pH} 2.0$ y CE $4.0 \mathrm{mS} / \mathrm{cm}$.

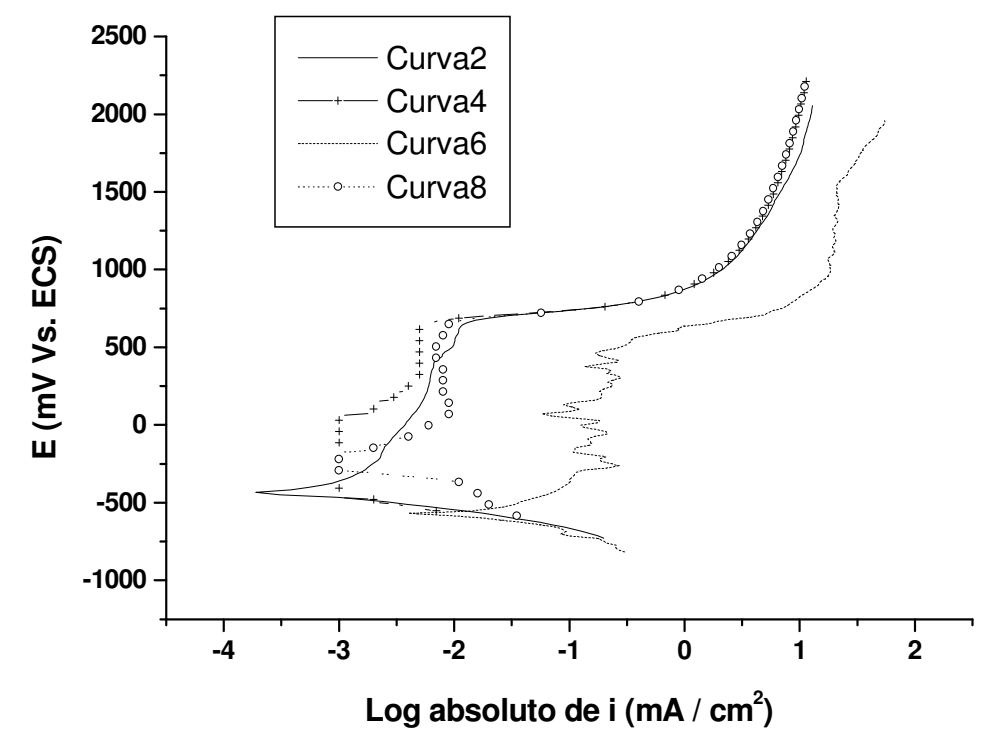

Gráfica 2. Curvas potenciodinámicas del diseño de experimentos $2^{4}$, bajo condiciones de $\mathrm{pH} 12$ y CE $4.0 \mathrm{mS} / \mathrm{cm}$.

Las curvas 2, 4, 6 y 8 de la gráfica 2, presentan un comportamiento de pasivación

- activación errático y este comportamiento es más pronunciado en las curvas 4 y 6, no presentando relación exclusiva con alguna de las variables, indicando que 
la formación de las capas pasivantes bajo condiciones de pH 12 y CE $4 \mathrm{mS} / \mathrm{cm}$, son inestables en un barrido potenciodinámico hasta los $750 \mathrm{mV}$, independientemente de DE y Ac. En potenciales superiores el comportamiento es más estable y no se observa pasivación. Analizando las curvas 4 y 8 vs. la curva 6 , se observa que la última de ellas presenta densidades de corriente mayores y se diferencia de las primeras en que presenta una menor distancia entre electrodos, indicando que posiblemente bajo condiciones de $\mathrm{pH} 12.0$ y $\mathrm{CE}$ $4.0 \mathrm{mS} / \mathrm{cm}$, se tengan mejores resultados a menores distancias entre electrodos independientemente del potencial, siempre y cuando no se haga presente la pasivación.

En la gráfica 3 se observa que el comportamiento de la densidad de corriente es estable y sin pasivación hasta los $1000 \mathrm{mV}$; posterior a este valor, la primera en presentar pasivación es la curva 13. El potencial de pasivación no guarda relación con la DE ni con Ac y por lo tanto se asume que la pasivación puede presentarse entre 1000 y $1500 \mathrm{mV}$ bajo condiciones de pH 2.0 y CE $45 \mathrm{mS} / \mathrm{cm}$; después de los $1500 \mathrm{mV}$ se presenta la reactivación. Así mismo, se puede considerar que bajo estas mismas condiciones, DE y Ac posiblemente no tendrán un efecto significativo en potenciales fuera del rango de pasivación.

Al igual que en la gráfica 2, en la gráfica 4 se observa que todas las curvas presentan comportamiento errático en la densidad de corriente y este comportamiento errático se presenta más pronunciado en las curvas 10 y 14. Aparentemente, bajo condiciones de $\mathrm{pH} 12$, y debido a las diferentes trayectorias de las curvas, todas las variables presentarán influencia en la densidad de corriente en los estudios potenciostáticos.

Las curvas de la gráfica 4 que presentaron mayor densidad de corriente al final del estudio son la 12 y 14 quienes nuevamente no guardan relación con DE ni con Ac. 


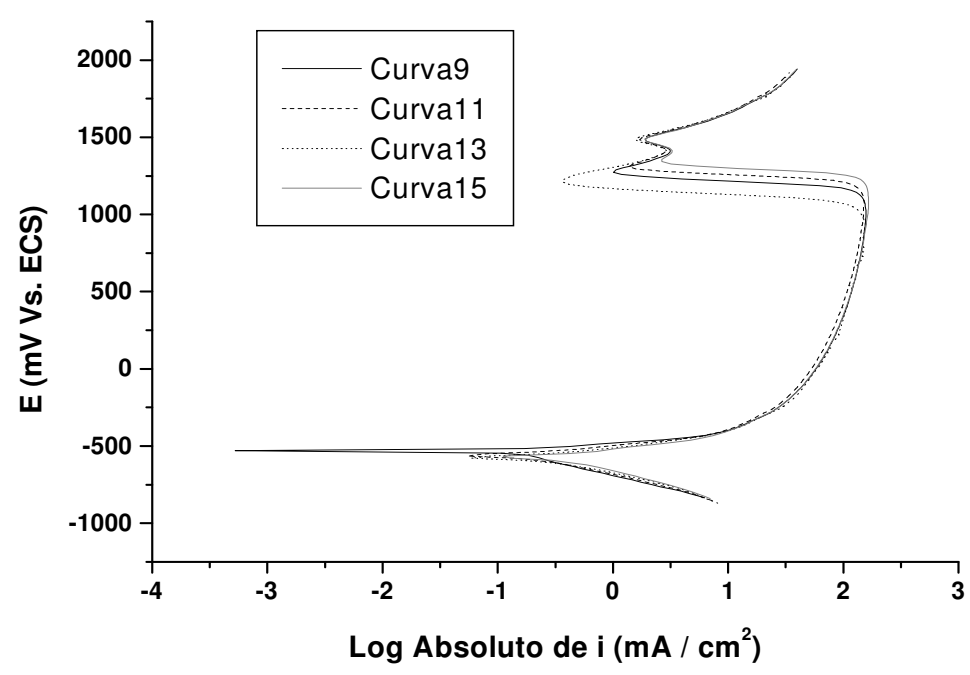

Gráfica 3. Curvas potenciodinámicas del diseño de experimentos $2^{4}$ bajo condiciones de $\mathrm{pH} 2.0$ y CE $45.0 \mathrm{mS} / \mathrm{cm}$.

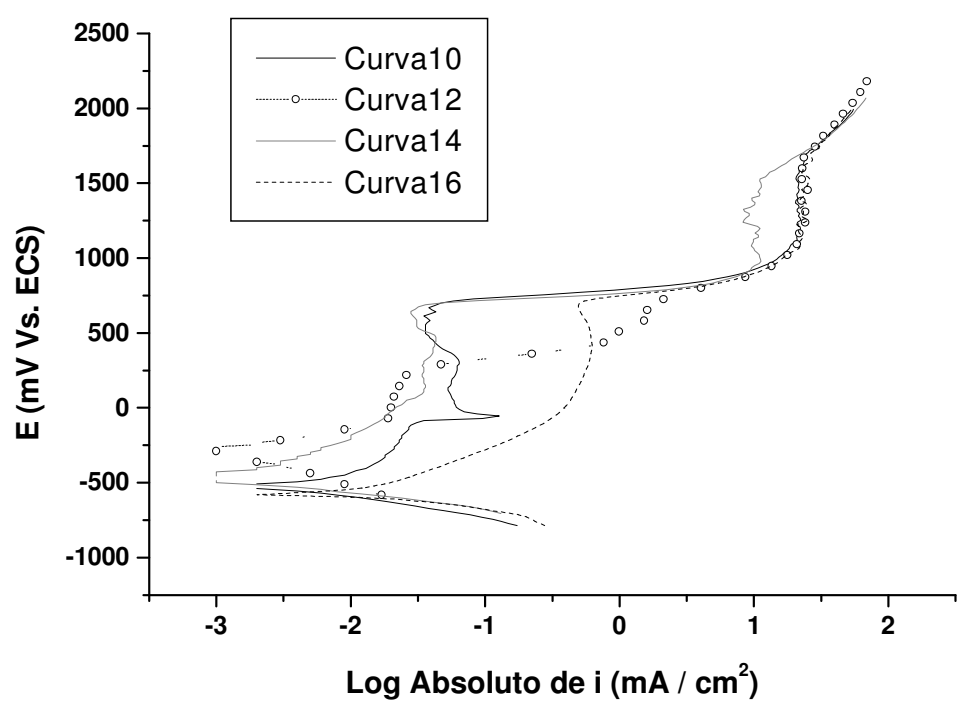

Gráfica 4. Curvas potenciodinámicas del diseño de experimentos $2^{4}$, bajo condiciones de $\mathrm{pH} 12.0$ y CE $45.0 \mathrm{mS} / \mathrm{cm}$.

Analizando en forma general las curvas de las gráficas 1 a 4 , se observa que a $\mathrm{pH}$ 2.0 se tiene un comportamiento más estable de la densidad de corriente; independientemente del resto de las variables de estudio y bajo estas condiciones de $\mathrm{pH}$, únicamente se tendrán riesgos de pasivación entre 1000 y $1500 \mathrm{mV}$ si el electrolito presenta conductividades eléctricas de $45 \mathrm{mS} / \mathrm{cm}$. En condiciones de $\mathrm{pH}$ 12, independientemente del resto de las variables de estudio, no se tiene 
certeza de los potenciales a los que se puede presentar o no la pasivación. Para los estudios potenciostáticos, se decidió seleccionar potenciales superiores a los $1800 \mathrm{mV}$ en donde se observa mayor estabilidad de las curvas.

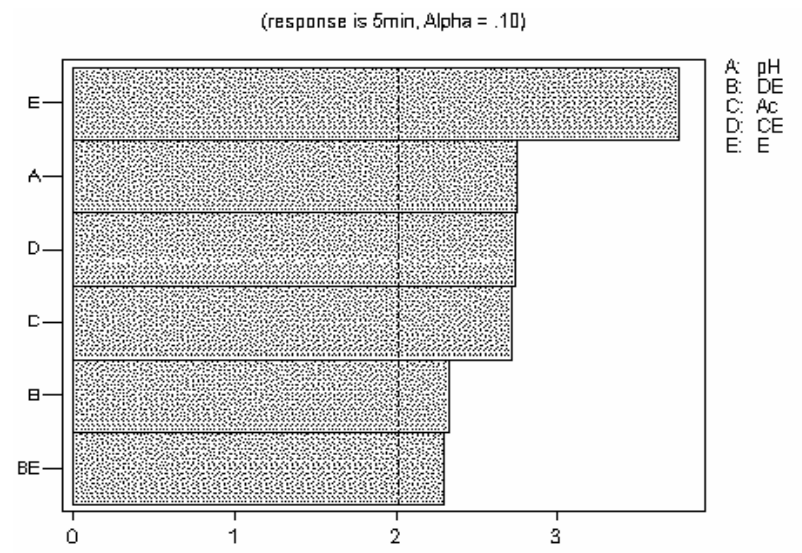

Grafica 5. Efectos absolutos de las variables sobre la densidad de corriente al minuto 5, donde la línea punteada representa el límite de significancia.

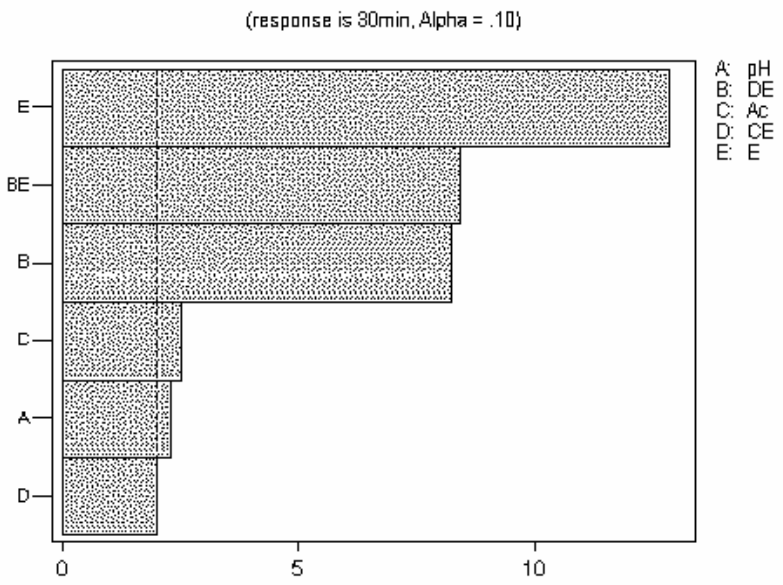

Gráfica 6. Efectos absolutos de las variables sobre la densidad de corriente al minuto 30, donde la línea punteada representa el límite de significancia.

Una vez obtenidos los resultados de las curvas potenciodinámicas se seleccionaron potenciales de $50 \mathrm{mV}$ y $2000 \mathrm{mV}$ para, mediante curvas potenciostáticas, estudiar el efecto cuantitativo de las variables sobre la densidad de corriente. 


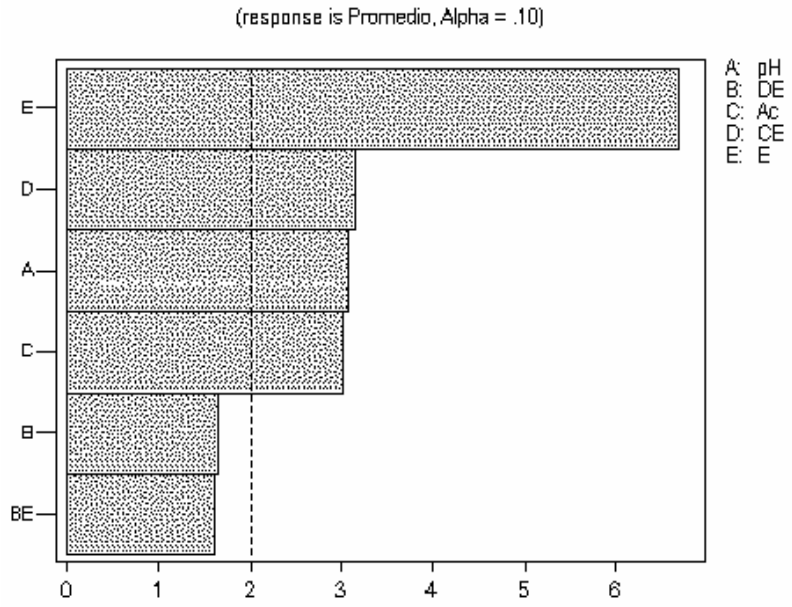

Gráfica 7. Efectos absolutos de las variables sobre la densidad de corriente a los 30 minutos, sustituyendo el valor de la curva 8 , minuto 30 , por el promedio de la densidad de corriente con el tiempo. La línea punteada representa el límite de significancia.

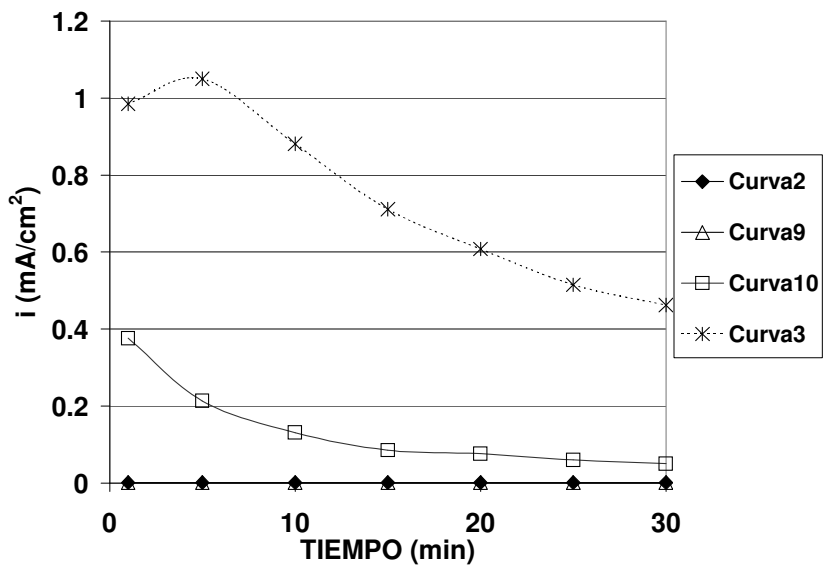

Gráfica 8. Comportamiento de la densidad de corriente con el tiempo de las curvas bajo potenciales de $50 \mathrm{mV}$ del diseño de experimentos $2^{5-2}$, en dos bloques y con puntos centrales. 


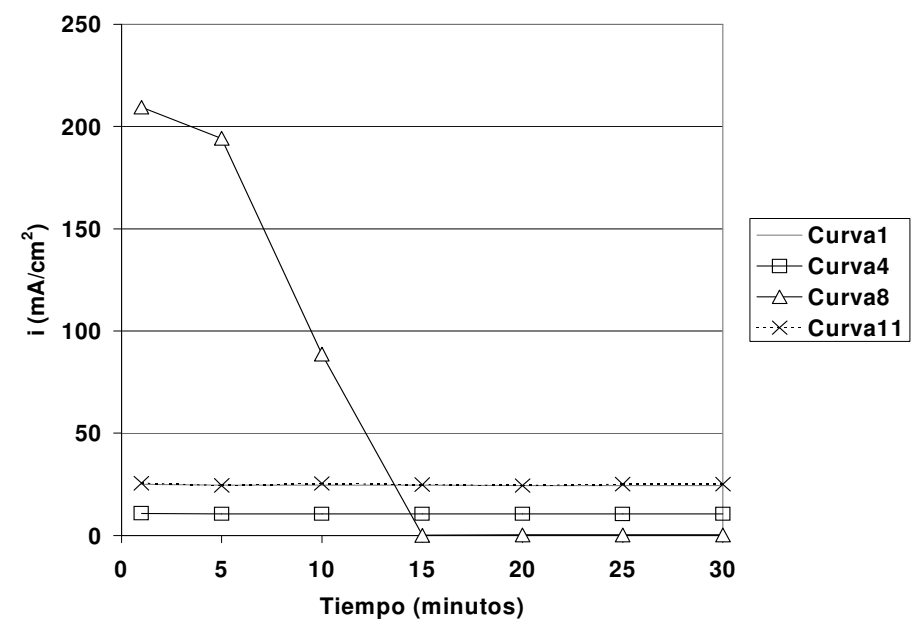

Gráfica 9. Comportamiento de la densidad de corriente con el tiempo de las curvas bajo potenciales de $2000 \mathrm{mV}$ del diseño de experimentos $2^{5-2}$, en dos bloques y con puntos centrales.

El diseño de experimentos $2^{5-2}$ en dos bloques y con puntos centrales, provee suficiente información para determinar si todas las variables de estudio tienen un efecto significativo, ya sea en forma individual o en interacción con otras. Así mismo, se pueden identificar las mejores condiciones para obtener la mayor densidad de corriente.

Como se observa en el cuadro 2, minuto 30, en las curvas 5 a 7 y 12 a 14, se obtienen mayores densidades de corriente, a excepción de la curva 8 que a los 5 minutos reporta densidades de corriente de $194.186 \mathrm{mV} / \mathrm{cm}^{2}$, pero posteriormente se pasiva llegando a valores cercanos a cero en el minuto 30. Los resultados obtenidos en las curvas primeramente señaladas, indican que existe un nivel óptimo para cada variable y este óptimo no se presenta en los niveles máximos.

El comportamiento general de las curvas presentadas en las gráficas 8 a 10 imposibilitan que un análisis estadístico sea válido para cualquier punto de la curva, incluso si se trata de condicionar la validez del análisis para los últimos 15 o 20 minutos donde los resultados son más estables; la pasivación presentada por la curva 8 de la gráfica 9 , traería como consecuencia una interpretación equivocada de los efectos de las variables, ya que las densidades de corriente no son las que se obtendrían si no se presenta la pasivación prácticamente total. 


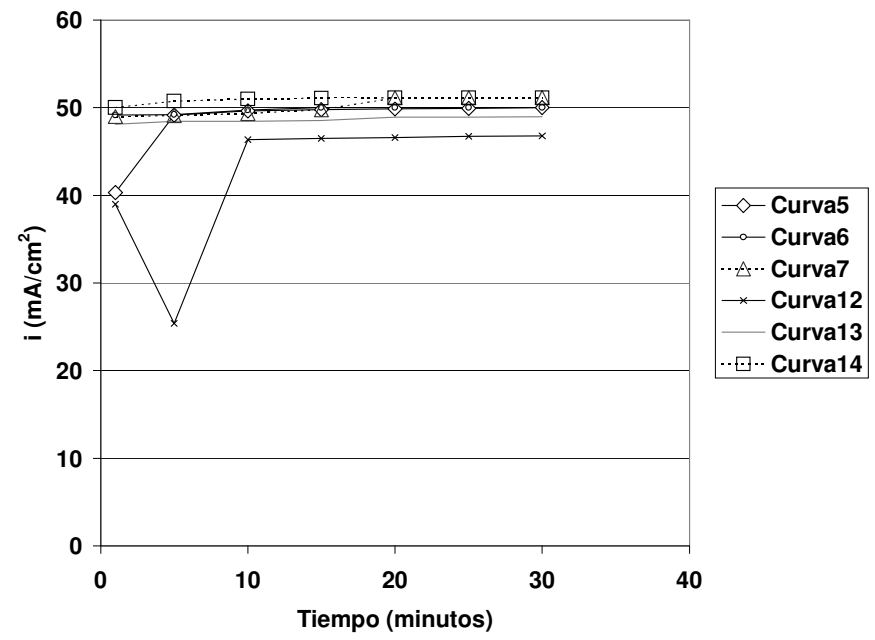

Gráfica 10. Comportamiento de la densidad de corriente con el tiempo de las curvas con nivel central de las variables del diseño de experimentos $25-2$, en dos bloques y con puntos centrales.

Para apreciar lo anterior, se presentan las gráficas 5 y 6 donde se muestran los efectos absolutos de las variables al analizar la densidad de corriente en el minuto 5 y en el minuto 30, respectivamente; se observa que en el minuto 5, todas las variables son significativas y en forma individual tienen más efecto que en interacción, mientras que en el minuto 30, únicamente el potencial (E) presenta mayor efecto que la interacción potencial - distancia entre electrodos; incluso la CE está en el límite de significancia.

Al realizar un análisis estadístico cambiando la densidad de corriente de la curva 8, de $0.228 \mathrm{~mA}$ al minuto 30, por una densidad de corriente de $70.429 \mathrm{~mA}$ que corresponde a la media aritmética en el tiempo, los efectos mostrados en la gráfica 7, indican que la distancia entre electrodos y la interacción potencialdistancia entre electrodos no son significativas. La diferencia en la significancia de las variables en cada uno de los análisis estadísticos muestran la importancia de la pasivación en la interpretación de resultados y la necesidad de identificar las condiciones de trabajo donde ésta no se presente para y así, la emisión de iones metálicos no sea significativamente diferente con el tiempo.

La variación en los resultados confirma que el análisis estadístico no es representativo para toda la superficie de las curvas; no obstante, como se muestra 
en el cuadro 3, el análisis de varianza al minuto 30 se observa que las variables e interacciones tienen efecto significativo en la emisión de iones metálicos y por lo tanto no deben dejar de considerarse en los estudios de electrocoagulación.

Cuadro 3. Análisis de varianza en el minuto 30 (unidades codificadas).

\begin{tabular}{|c|c|c|c|c|c|c|}
\hline Source & DF & Seq SS & Adj SS & Adj MS & F & P \\
\hline Blocks & 1 & 12.42 & 12.42 & 12.42 & 4.62 & 0.084 \\
\hline Main Effects & 5 & 667.74 & 667.74 & 133.55 & 49.70 & 0.000 \\
\hline $\begin{array}{c}\text { 2- Way } \\
\text { Interactions }\end{array}$ & 1 & 190.62 & 190.62 & 190.62 & 70.94 & 0.000 \\
\hline Curvature & 1 & 6029.69 & 6029.69 & 6029.69 & 2000 & 0.000 \\
\hline Residual Error & 5 & 13.44 & 13.44 & 2.69 & & \\
\hline Lack of Fit & 1 & 1.66 & 1.66 & 1.66 & 0.56 & 0.495 \\
\hline Pure Error & 4 & 11.78 & 11.78 & 2.94 & & \\
\hline Total & 13 & 6913.91 & & & & \\
\hline
\end{tabular}

Dado que el análisis estadístico no resultó recomendable para tomar decisiones sobre las condiciones en las cuales se obtienen las mejores densidades de corriente, es necesario realizar un análisis cualitativo de las curvas potenciostáticas.

En la gráfica 8, se aprecia que todas las curvas se pasivan o tienden hacia la pasivación indicando que potenciales de $50 \mathrm{mV}$, independientemente de las otras variables, no son recomendables para la producción de iones metálicos. Las curvas potenciodinámicas indicaron que sólo en $\mathrm{pH} 12$ se tendría riesgo de pasivación, sin embargo, el comportamiento de las curvas 3 y 10 que se realizaron bajo condiciones de $\mathrm{pH} 2$, muestra que el tiempo es de amplia influencia en la formación de capas pasivas. Las mejores densidades de corriente se presentan en la curva 3 y aparentemente no guardan relación con alguna variable en específico.

En la gráfica 9 se observa que solo la curva 8 presenta pasivación aún y cuando inicia con densidad de corriente hasta 10 veces mayor que las otras curvas. Las curvas 1 y 11 presentan densidades de corriente prácticamente iguales y coinciden en que se realizaron a $2000 \mathrm{mV}$ y DE de $14 \mathrm{~cm}$, indicando que bajo esas condiciones el $\mathrm{pH}$, Ac y DE posiblemente no tengan influencia en los resultados. 
Las curvas de la gráfica 10 corresponden al nivel central de las variables; las densidades de corriente al minuto 30, presentan una media de $49.68 \mathrm{~mA}$ con una desviación estándar de 1.63; sin embargo, al realizar estos cálculos estadísticos en el minuto 5, donde se observa la mayor diferencia entre densidades de corriente, la densidad de corriente media es de $45.36 \mathrm{~mA}$ con una desviación estándar de 9.8 indicando que los resultados en ese punto no tienen buena repetibilidad. Se observa en ésta misma gráfica que la curva 12, a diferencia de las otras curvas, presenta pasivación y reactivación, no obstante que al reactivarse no alcanza los valores de densidades de corriente obtenidas por las otras curvas, indicando la posible presencia de una capa pasivante no continua. La curva 5 inicia, también, con problemas de pasivación pero se reactiva en el primer minuto alcanzando densidades de corriente superiores a la media. La diferencia en el comportamiento de estas curvas, indica que la formación y permanencia de capas pasivantes puede ser influenciada por otros factores además de los considerados en este estudio.

Las mayores y más estables densidades de corriente se obtienen bajo el nivel central de todas las variables, lo cual indica que se tendrán mayores emisiones de iones metálicos en estas condiciones y dado que la coagulación floculación, de la mayoría de los contaminantes del agua, se presenta en un rango de $\mathrm{pH}$ de ligeramente ácido a ligeramente alcalino, seleccionar estos parámetros para un reactor de electrocoagulación pudiera resultar en mejores eficiencias de remoción, que bajo cualquiera otra de las condiciones estudiadas.

\section{Conclusiones de las curvas potenciodinámicas}

- Las curvas potenciodinámicas no pueden ser consideradas como único medio para determinar la presencia o ausencia de la pasivación bajo diferentes condiciones de estudio.

- A pH 12 se presentan más riesgos de pasivación, no obstante, a pH 2 y CE de $40 \mathrm{mS} / \mathrm{cm}$, se puede también presentar la pasivación a potenciales superiores de $1000 \mathrm{mV}$ y menores a $1750 \mathrm{mV}$. 
- El efecto de las variables de estudio es más representativo bajo condiciones de $\mathrm{pH} 12$.

- El análisis estadístico no es válido para todos los puntos de las curvas y por lo tanto el análisis cualitativo es una buena opción para observar el efecto de las variables.

Cuadro 4. Tabla de abreviaturas utilizadas en este estudio.

\begin{tabular}{|c|c|}
\hline Literales & Significado \\
\hline $\mathrm{E}$ & Potencial $(\mathrm{mV})$ respecto al electrodo calomelano saturado \\
\hline $\mathrm{ECS}$ & Electrodo Calomelano Saturado \\
\hline $\mathrm{i}$ & Densidad de corriente $\left(\mathrm{mA} / \mathrm{cm}^{2}\right)$ \\
\hline $\mathrm{DE}$ & Distancia entre electrodos $(\mathrm{cm})$ \\
\hline $\mathrm{CE}$ & Conductividad eléctrica $(\mathrm{mS} / \mathrm{cm})$ \\
\hline $\mathrm{Ac}$ & Área de electrodo auxiliar $\left(\mathrm{cm}^{2}\right)$ \\
\hline
\end{tabular}

\section{Conclusiones de las curvas potenciostáticas}

- La pasivación se presenta a potenciales de $50 \mathrm{mV}$ independientemente del resto de las variables; a potenciales de $2000 \mathrm{mV}$ sólo se presenta cuando se tiene $\mathrm{pH} 2$ y altas conductividades eléctricas.

- Las variables seleccionadas no son las únicas que influyen en la formación y permanencia de las capas pasivantes.

- El tiempo es un factor importante en la formación de las capas pasivantes.

- La pasivación es una variable que al influenciar la emisión de iones metálicos puede influenciar a las eficiencias de remoción de contaminantes en pruebas con reactores de electrocoagulación.

- Bajo el nivel central de todas las variables se obtienen mayores densidades de corriente. 


\section{Pasivación de los Electrodos en el Proceso de Electrocoagulación}

e-mails: gilberto.badillo@cimav.edu.mx, facundo.almeraya@cimav.edu.mx, teresa.alarcon@cimav.edu.mx

Resumen

Una de las principales limitantes de la electrocoagulación como proceso de tratamiento para remoción de contaminantes del agua residual, es el desconocimiento del grado de interacción de algunas variables que podrían influir significativamente al sistema, de las cuales, la pasivación de los electrodos es una de ellas, debido a que su presencia limita la emisión de iones metálicos y por consecuencia se incrementan los tiempos de tratamiento, costos de operación y se reduce la eficiencia de remoción. En el presente estudio se emplearon curvas potenciodinámicas y potenciostáticas para observar el comportamiento de la densidad de corriente ante el efecto del $\mathrm{pH}$, conductividad eléctrica, distancia entre electrodos, área de electrodo auxiliar y la diferencia de potencial aplicado, determinándose que todas las variables tienen influencia en la densidad de corriente; que la pasivación se presenta a potenciales de $50 \mathrm{mV}$, independientemente del resto de las variables, y que a potenciales de $2000 \mathrm{mV}$, sólo se presenta cuando se tiene $\mathrm{pH} 2$ y altas conductividades eléctricas.

Palabras clave: curvas potenciodinámicas y potenciostáticas, densidad de corriente, agua residual.

\section{Referencias}

1. L. Joffe and L. Kniper, Industrial Waste Water 20 (2000) 1-6.

2. F. Chuanping, S. Norio, Sh. Satoru, M. Takaaki, J. Haz. Mater. B 103 (2003) 65-78.

3. M.Y. Mollah, R. Shennach, J.R. Praga, D.L. Cocke, J. Haz. Mater. B 84 (2001) 29-41.

4. C. Tsouris, D.W. DePaoli, J.T. Shor, M.Z.-C. Hu, T.Y. Ying, Colloids Surf. Phys. Eng. Asp. 177 (2001) 223-233.

5. N. Mameri, H. Lounici, D. Belhocine, H. Grib, D.L. Piron, Y. Yahiat, Separat. Purif. Tech. 24 (2001) 113-119.

6. U.R. Evans, “Corrosiones metálicas". Editoriales Reverte, S.A de C.V. (1987). pp. 68-79.

7. F.J.A. González, “Control de la corrosión. Estudio y medida por técnicas electroquímicas”. Editorial C.S.I.C. España. (1989). pp. 165-193. 
8. X. Chen, G. Chen and P.L. Yue, Separat. Purif. Tech. 19 (2000) 65-76.

9. G. Chen, X. Chen, P.L. Yue, J. Env. Eng. 126-9 (2000) 858-863. 